\title{
Diabetes drugs for nonalcoholic fatty liver disease: a systematic review
}

\author{
Ian Blazina ${ }^{*}$ (D) and Shelley Selph
}

\begin{abstract}
Background: Fatty liver is associated with obesity, type 2 diabetes, hyperlipidemia, hypertension, and metabolic syndrome. While there are no approved drugs for the treatment of nonalcoholic fatty liver disease (NAFLD) or nonalcoholic steatohepatitis, strategies to ameliorate fatty liver often target these related diseases. We sought to determine if any medications approved by the US Food and Drug Administration to treat diabetes are helpful in reducing weight and improving steatohepatitis in patients with NAFLD.

Methods: We conducted a systematic review of published and unpublished studies evaluating the comparative effectiveness and harms of diabetes medications for the treatment of NAFLD. We searched MEDLINE, EMBASE, Cochrane Database of Systematic Reviews, and the Cochrane Central Register of Controlled Trials through 3rd quarter, 2019 using terms for included drugs and indications.
\end{abstract}

Results: We screened 1591 citations and included 18 trials of diabetes drugs to treat NAFLD. Studies of metformin found no difference from placebo in steatosis, fibrosis, NAFLD activity score, or resolution of NASH. While weight and glucose control were improved with metformin, it did not substantially impact liver disease. Studies of pioglitazone in NASH patients found benefits in liver function, liver fat, and NASH resolution, though significant increases in weight may be cause for concern. Evidence for other thiazolinediones was more limited and had somewhat mixed results, but findings were generally consistent with those for pioglitazone: liver fat and function and glucose measures improved, but weight also increased. We found some evidence that liraglutide improves liver fat, liver function, and HbA1c and is effective at resolving NASH and reducing weight. Exenatide performed less well but also resulted in significant reductions in liver fat and weight.

Conclusions: Consistent with existing clinical practice guidelines, which recommend lifestyle intervention and treatment for comorbidities related to fatty liver disease as first-line treatment, trial evidence supports the efficacy of some diabetes drugs (especially pioglitazone) in patients with NAFLD or NASH, though weight gain with some diabetes drugs may warrant caution. Larger trials are needed to better characterize the efficacy and harms of diabetes pharmacotherapy in these patients.

Keywords: Systematic review, Nonalcoholic fatty liver disease, Diabetes, Pharmacotherapy

\section{Background}

Nonalcoholic fatty liver disease (NAFLD), the accumulation of excess fat in the liver (steatosis) not resulting from excessive alcohol consumption or another secondary cause, is a growing public health issue associated with the global epidemics of obesity and type 2 diabetes [1]. NAFLD represents a spectrum of diseases, from mild steatosis to nonalcoholic steatohepatitis (NASH) and

\footnotetext{
* Correspondence: blazina@ohsu.edu

Pacific Northwest Evidence-based Practice Center, Oregon Health \& Science

University, 3181 SW Sam Jackson Park Rd, Mailcode: BICC, Portland, OR

97239, USA
}

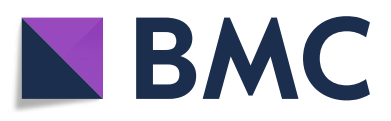

(c) The Author(s). 2019 Open Access This article is distributed under the terms of the Creative Commons Attribution 4.0 International License (http://creativecommons.org/licenses/by/4.0/), which permits unrestricted use, distribution, and reproduction in any medium, provided you give appropriate credit to the original author(s) and the source, provide a link to the Creative Commons license, and indicate if changes were made. The Creative Commons Public Domain Dedication waiver (http://creativecommons.org/publicdomain/zero/1.0/) applies to the data made available in this article, unless otherwise stated. is estimated to be $20 \%$ to $30 \%$, with around 2 to $3 \%$ of the population having NASH [2]. NAFLD is a common cause of cirrhosis, end-stage chronic liver disease, liver transplantation, and hepatocellular carcinoma [3]; liverrelated mortality is about twice as high among those with NAFLD than those without [2].

Fatty liver is associated with obesity, type 2 diabetes, hyperlipidemia, hypertension, and metabolic syndrome. While there are no approved drugs for the treatment of 
NAFLD or NASH, strategies to ameliorate fatty liver often target these related diseases [4].

We performed a systematic review to determine if any medications approved by the US Food and Drug Administration (FDA) to treat diabetes are helpful in reducing weight and improving steatohepatitis in patients with NAFLD in the setting of many new drugs in development seeking an indication for NAFLD or NASH. The original review, which was commissioned by the Drug Effectiveness Review Project and is not publicly available, also evaluated the success of weight loss drugs, dyslipidemia drugs, diet, and exercise in weight loss and improvement of NAFLD. Here, we focus on the evidence for diabetes medications, which was the most well-studied intervention area.

We sought evidence to answer the following questions:

1. What is the comparative efficacy and effectiveness of FDA-approved drugs that are used off-label to treat nonalcoholic fatty liver disease?

2. What are the comparative harms of FDA-approved drugs that are used off-label to treat nonalcoholic fatty liver disease?

\section{Methods}

We followed systematic review methodology and procedures developed specifically for the Drug Effectiveness Review Project (DERP) [5] and that are in accordance with current guidance for systematic reviews.

\section{Data sources and searches}

We searched MEDLINE, EMBASE, Cochrane Database of Systematic Reviews, and the Cochrane Central Register of Controlled Trials through 3rd quarter, 2019, using terms for included drugs and indications (Additional file 1). We consulted medical reviews from the Food and Drug Administration's Center for Drug Evaluation and Research and requested additional unpublished trial data from relevant pharmaceutical companies.

\section{Study selection}

Eligible studies were head-to-head or placebo-controlled randomized controlled studies of adults with nonalcoholic fatty liver disease (including NASH) who received an FDA-approved diabetes drug to treat NASH/NAFLD. We excluded any drug developed specifically for the treatment of NAFLD, including the FDA-approved obeticholic acid as well as drugs in development specifically for treatment of NALFD (selonsertib, elafibranor, and cenicriviroc), as well as studies that only evaluated different doses of the same drug (dose-ranging studies). Benefit outcomes of interest included changes in alanine aminotransferase test (ALT), aspartate aminotransferase test (AST), liver fat, liver fibrosis, and resolution of NAFLD; weight loss (e.g., pounds lost, change in BMI, loss of $10 \%$ body weight); long-term health outcomes (e.g., mortality, need for liver transplant); and HbA1c or other glucose outcomes. Relevant harms included serious adverse events and withdrawals due to adverse events. We required studies to have at least 30 patients per treatment arms of interest unless the trial included liver histology, the gold standard, at the conclusion of the trial; in which case, we reduced the required sample size to 20 per treatment arm.

One reviewer screened citations and a second reviewer assessed excluded citations. Two reviewers independently evaluated full-text articles by applying the inclusion criteria and resolved disagreements by consensus.

\section{Data abstraction and quality assessment}

Information on population characteristics, interventions, subject enrollment and discontinuation, and results for effectiveness and harm outcomes were abstract by one reviewer. The second reviewer verified abstracted data.

Study quality was assessed independently by two reviewers according to the DERP's methods [5], focusing on methods of randomization, allocation concealment, blinding of providers, outcome assessors, and patients; similarity of group characteristics at baseline, especially of prognostic factors; attrition rate; and the use of intent-to-treat analysis. Studies that met all criteria were rated as good quality; studies with an element at high risk of bias or failed to meet combinations of criteria were rated as poor quality; and the remaining studies were rated fair quality. Disagreements between reviewers were resolved by consensus.

\section{Grading strength of evidence}

We graded strength of evidence according to the Agency for Healthcare Research and Quality's (AHRQ) guidance for the Evidence-based Practice Center Program [6]. Similar to the GRADE method, this approach assesses risk of bias, consistency, directness, and precision of the evidence. Strength of evidence was graded for key outcome measures of liver fat, weight change, and ALT or AST elevations. Grades reflect the strength of the body of evidence to answer key questions on the effectiveness and harms of included drugs, not general efficacy of the drugs. Two reviewers independently assessed each domain for each outcome and differences were resolved by consensus.

\section{Results}

We screened 1591 citations and included 39 trials (in 41 publications) in the primary report; here, we report only the evidence pertaining to diabetes drugs (18 trials in 17 publications) [7-22] (Fig. 1). Most trials were small $(N<100$ per treatment arm) and rated fair quality, primarily due to unclear blinding, unclear allocation concealment, and high attrition. Five trials were rated good quality $[7,9,18,19,22]$. Some trials enrolled patients at 


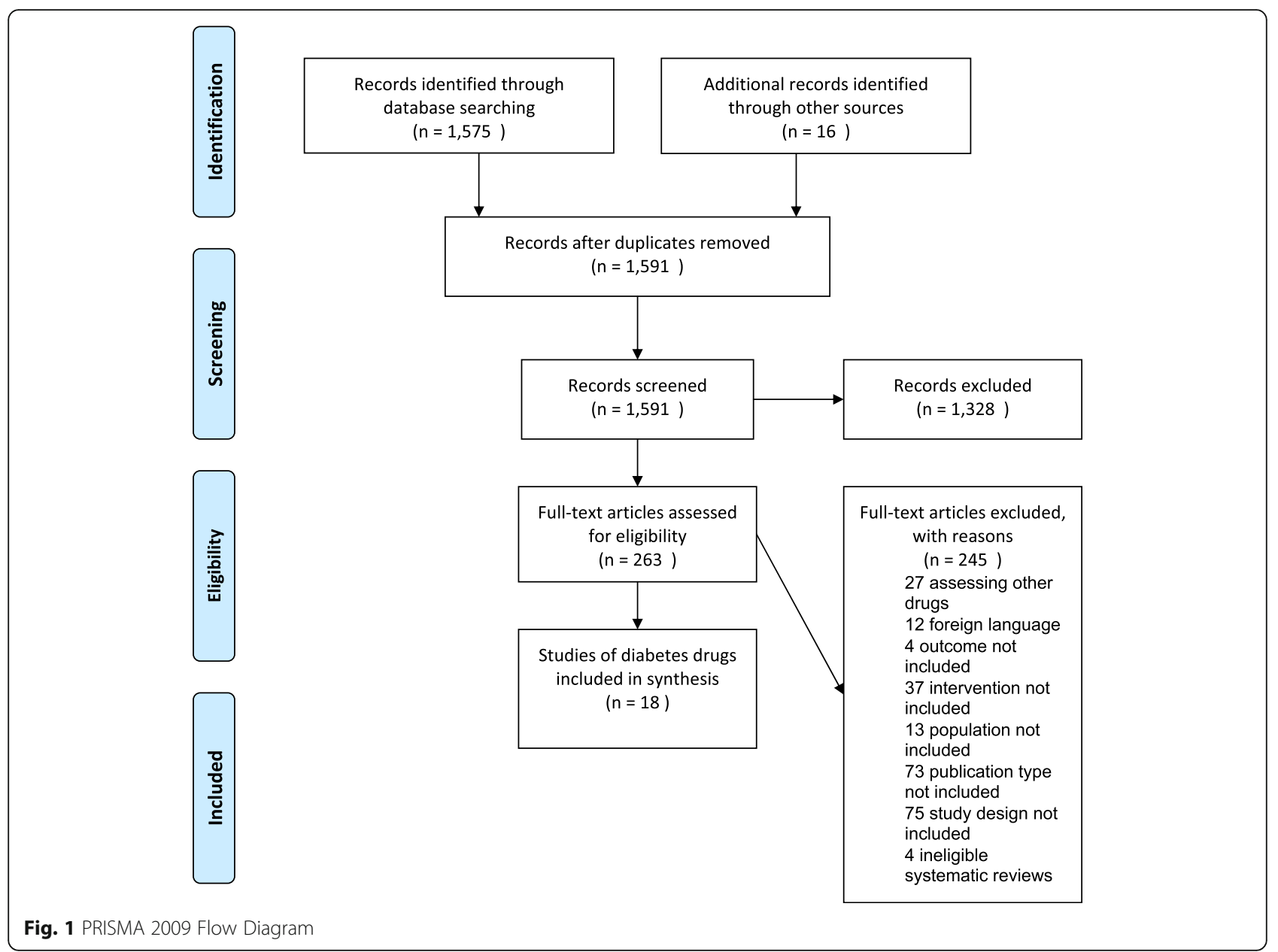

the more severe end of NAFLD continuum who were diagnosed with $\mathrm{NASH}$, and some trials required enrollees to also have metabolic syndrome, prediabetes, type 2 diabetes, hypertension, and/or be overweight or obese.

Twelve individual randomized trials in NAFLD patients with $[7,8]$ and without $[9-17,22,23]$ type 2 diabetes included treatment with a diabetes drug in 1 or more treatment arms. In addition, 3 trials enrolled patients with prediabetes or type 2 diabetes [18-20]. We [23] also included an individual patient data (IPD) metaanalysis of 6 RCTs of liraglutide in patients with diabetes [21]. Most studies did not report harm outcomes. Ten studies evaluated thiazolidinediones (Table 1), three studies evaluated GLP-1 agonists (Table 2), six studies evaluated metformin (Table 3), and one study evaluated a DPP-4 inhibitor (Table 4).

\section{Patients with NAFLD and diabetes Liraglutide}

An IPD meta-analysis of 6 RCTs $(N=3258)$ [21] and 2 additional, unique trials $[7,8](N=132)$ enrolled
NAFLD patients with type 2 diabetes. All RCTs in the IPD meta-analysis were 26-week trials and treated patients with liraglutide $0.6 \mathrm{mg}$, liraglutide $1.2 \mathrm{mg}$, liraglutide $1.8 \mathrm{mg}$, or placebo. The IPD meta-analysis found that in patients with an elevated ALT $(N=1387)$, liraglutide $1.8 \mathrm{mg}$ reduced ALT to a significantly greater degree than placebo, but the effect was lost after correcting for patient weight loss $(P=0.21)$ or after correcting for improvement in $\mathrm{HbA1c}(P=0.63)$. Findings were similar for decrease in hepatic fat. In a sub-study $(n=149)$, there was a trend for reduction in liver fat (measured with CT scanning) with liraglutide $1.8 \mathrm{mg}(P=0.07)$, but the effect was lost after adjusting for weight loss $(P=$ $0.90)$ or HbA1c $(P=0.73)$. While the incidence of serious adverse events was similar between liraglutide and placebo $(6.5 \%$ vs. $6 \%$, RR $1.10,95 \%$ CI 0.75 to 1.62), study withdrawal due to adverse events and gastrointestinal disorder was significantly more likely in patients treated with liraglutide (withdrawal due to adverse events: $9 \%$ vs. $3 \%$, RR $3.59,95 \%$ CI 2.07 to 6.24; GI disorders $45 \%$ vs. $18 \%$, RR 2.54, $95 \%$ CI 2.10 to 3.08$)$. 


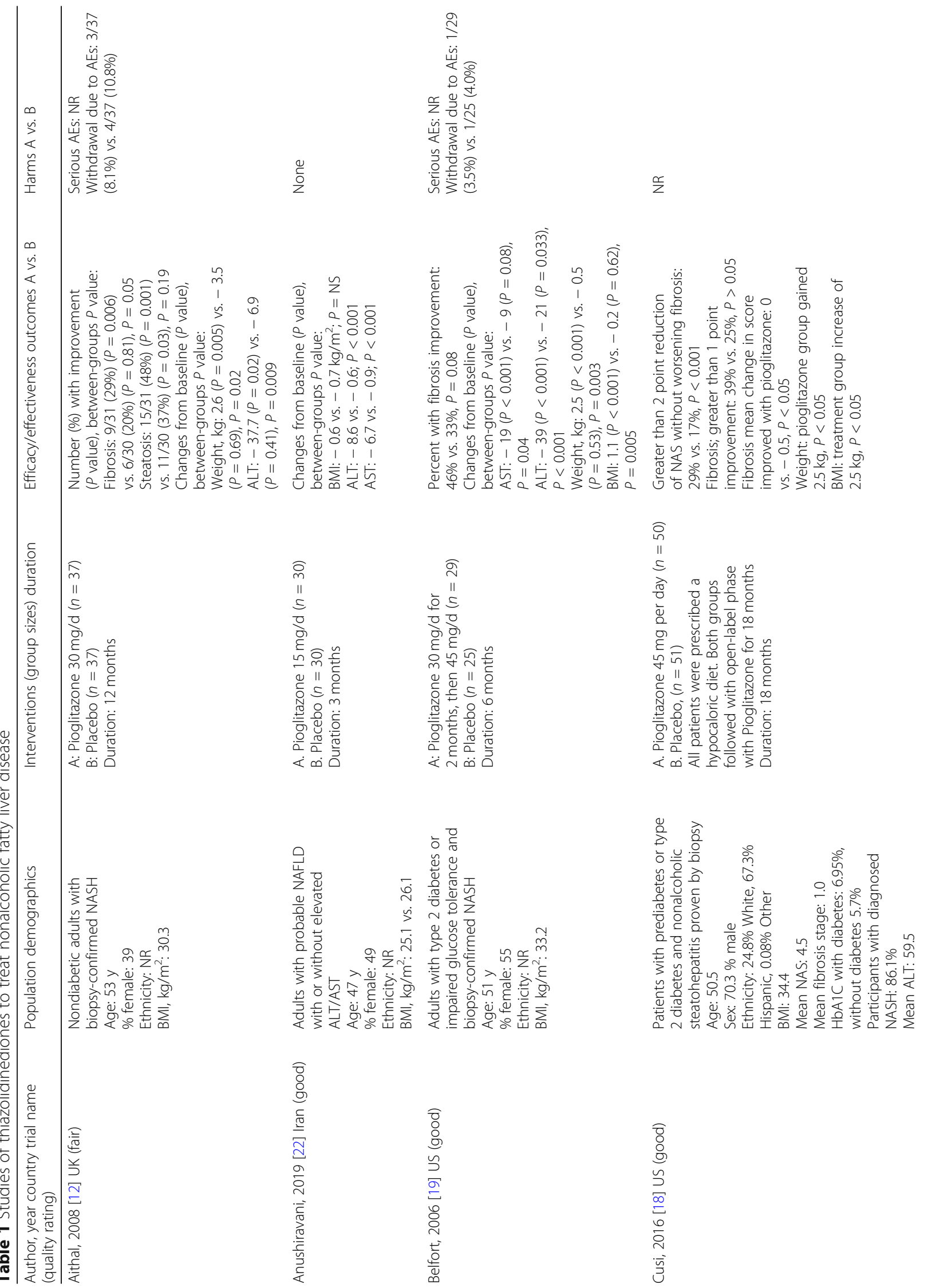




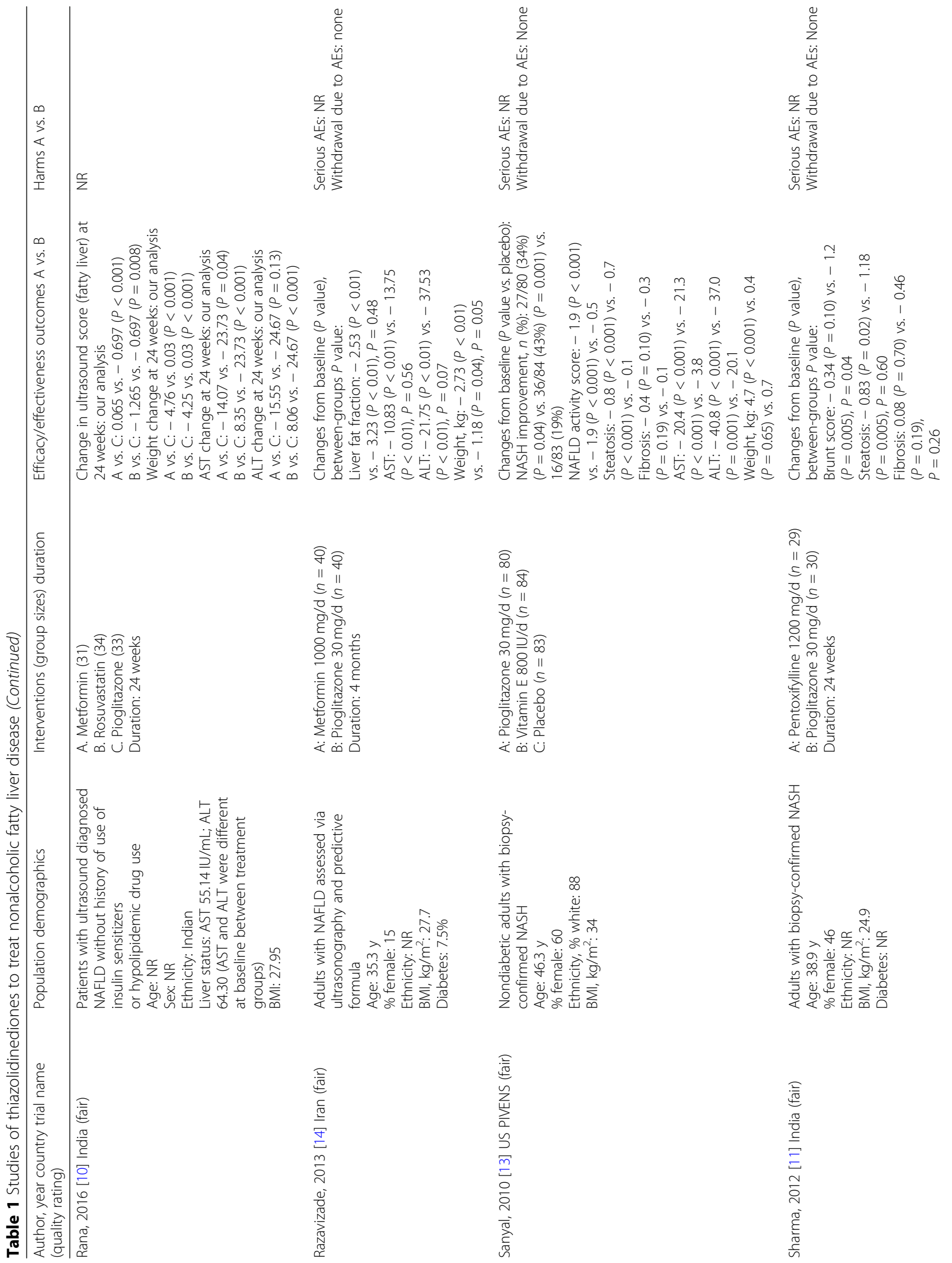




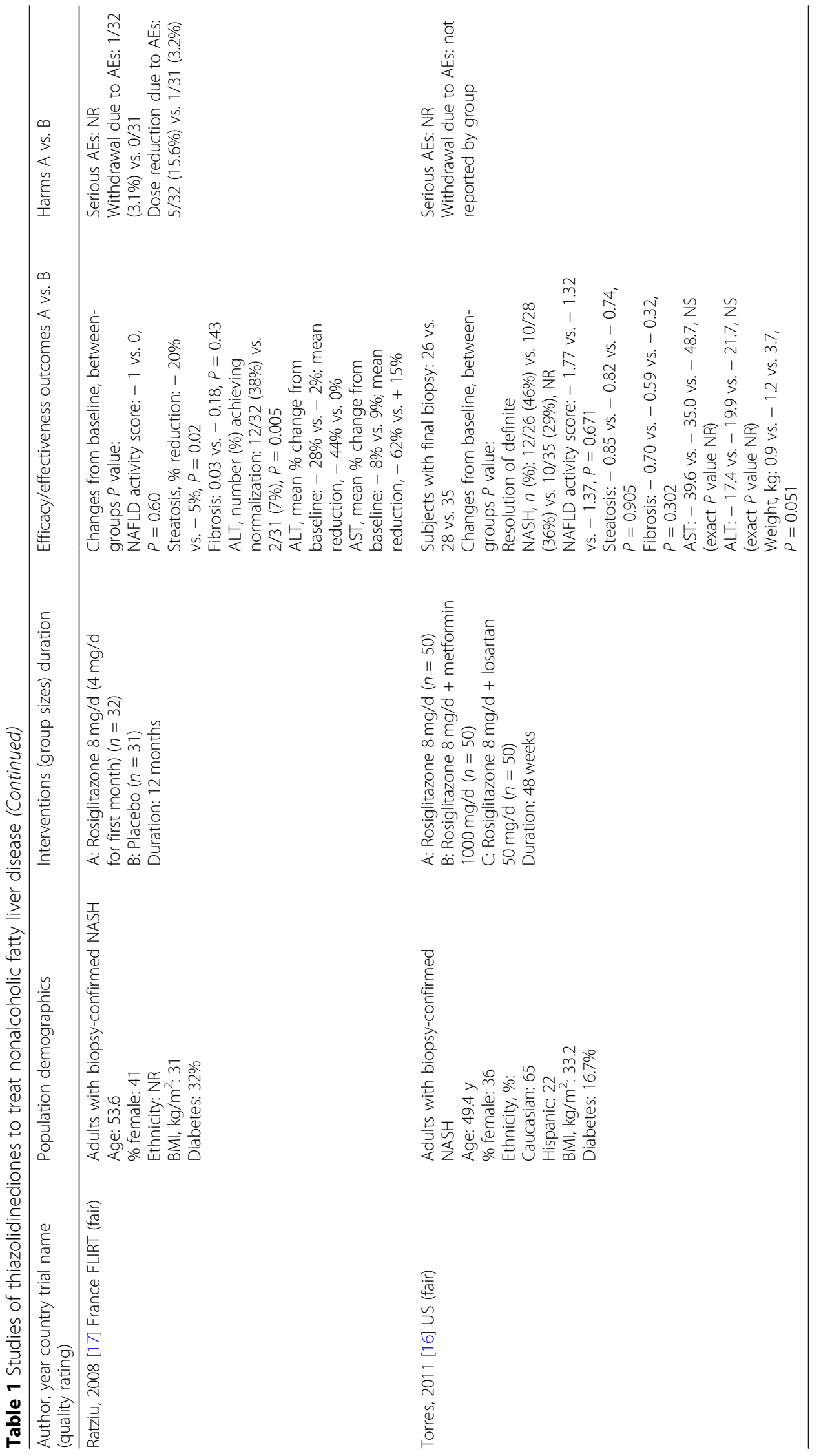


Table 2 Studies of GLP-1 agonists to treat nonalcoholic fatty liver disease

\begin{tabular}{|c|c|c|c|c|}
\hline $\begin{array}{l}\text { Author, year country trial } \\
\text { name (quality rating) }\end{array}$ & Population demographics & $\begin{array}{l}\text { Interventions (group sizes) } \\
\text { duration }\end{array}$ & $\begin{array}{l}\text { Efficacy/effectiveness } \\
\text { outcomes A vs. B }\end{array}$ & Harms A vs. B \\
\hline $\begin{array}{l}\text { Armstrong, } 2013 \text { [21] } \\
\text { Multinational LEAD } \\
\text { \& LEAD-2 (fair) }\end{array}$ & $\begin{array}{l}\text { Patients with type } 2 \\
\text { diabetes who were } \\
\text { unable to maintain } \\
\text { glycemic control } \\
\text { (HbA1c } \geq 7 \% \text { ) with } \\
\text { diet and exercise } \\
\text { alone, or with oral } \\
\text { antidiabetic treatment } \\
\text { Age: } 55.9 \text { years } \\
\text { Gender, \% } \\
\text { Female: } 46.5 \\
\text { Ethnicity, \%: } \\
\text { White: 78.6 } \\
\text { Asian/Hawaiian/Pacific } \\
\text { Islander: } 12.7 \\
\text { Black/African American: } 5.8 \\
\text { Other: 2.1 } \\
\text { BMI: NR } \\
\text { NAFLD/NASH Stage: NR } \\
\text { Liver enzymes (IU/L): } \\
\text { Total ALT mean (SD): } \\
\text { 29.4 (16.6) } \\
\text { Normal ALT mean (SD): } \\
\text { 19.1 (5.6) } \\
\text { Abnormal ALT mean (SD): } \\
\text { 39.4 (17.7) }\end{array}$ & $\begin{array}{l}\text { LEAD } \\
\text { A: Liraglutide } 0.6 \mathrm{mg} / \text { day }(475) \\
\text { B: Liraglutide } 1.2 \mathrm{mg} / \text { day (896) } \\
\text { C: Liraglutide } 1.8 \mathrm{mg} / \text { day (1363) } \\
\text { D: Placebo (524) } \\
\text { Duration: } 26 \text { weeks } \\
\text { LEAD-2 } \\
0.6,1.2, \text { or } 1.8 \mathrm{mg} / \text { day liraglutide, } \\
4 \mathrm{mg} / \text { day glimepiride or placebo, } \\
\text { all in combination } \\
\text { with metformin }\end{array}$ & $\begin{array}{l}\text { LEAD } \\
\text { In patients with elevated } \\
\text { ALT, liraglutide } 1.8 \\
\text { significantly reduced ALT } \\
\text { compared with placebo } \\
\text { (and was dose responsive); } \\
\text { however, after correcting } \\
\text { for change in weight, the } \\
\text { difference was no longer } \\
\text { significant: Mean } \\
\text { difference }-1.41, P=0.21 \text {, } \\
\text { with similar finding after } \\
\text { correcting for reduction in } \\
\text { HbA1c: Mean difference } 0.57 \text {, } \\
P=0.63 \text {. Lower doses of } \\
\text { liraglutide had similar effects } \\
\text { as placebo } \\
\text { LEAD2 } \\
64 \% \text { of patients had elevated } \\
\text { liver fat on CT; as above } \\
\text { liraglutide improved liver fat } \\
\text { in a dose dependent way; } \\
\text { however, there was no } \\
\text { significant differences } \\
\text { between liraglutide and } \\
\text { placebo after correcting } \\
\text { either for weight loss or } \\
\text { HbA1c ( } P=0.90 \text { and } 0.73 \text {, } \\
\text { respectively) }\end{array}$ & $\begin{array}{l}\text { LEAD } \\
\text { WAE: } 9 \% \text { vs. } 9 \% \text { vs. } 3 \% \\
\text { (liraglutide vs. placebo, } \\
P<0.001 \text { ) } \\
\text { SAE: } 7 \% \text { vs. } 6 \% \text { vs. } 6 \% \\
\text { Gl disorders: } 46 \% \text { vs. } 45 \% \\
\text { vs. } 18 \% \text { (liraglutide vs. } \\
\text { placebo, } P<0.001 \text { ) } \\
\text { LEAD2 } \\
\text { NR }\end{array}$ \\
\hline $\begin{array}{l}\text { Armstrong, } 2016 \text { [9] } \\
\text { UK } \\
\text { LEAN (good) }\end{array}$ & $\begin{array}{l}\text { Patients had histologically } \\
\text { confirmed NASH } \\
\text { Age: } 51 \\
\text { Sex: } 60 \% \text { male } \\
\text { Ethnicity: } \\
\text { White: } 88 \% \\
\text { Asian: } 4 \% \\
\text { Black: } 2 \% \\
\text { Other: } 6 \% \\
\text { Liver status: NAS: 4.9; } \\
\text { ALT: } 72 \text { IU/mL; F3-F4: 52\% } \\
\text { BMl: } 36 \\
\text { Diabetes: } 33 \%\end{array}$ & $\begin{array}{l}\text { A. Liraglutide } 1.8 \text { mg (26) } \\
\text { B. Placebo (26) } \\
\text { Duration: } 48 \text { weeks }\end{array}$ & $\begin{array}{l}\text { Resolution of NASH: } 39 \% \\
\text { vs. } 9 \% \text { (RR } 4.3,95 \% \mathrm{Cl} 1.0 \\
\text { to } 17.7 \text { ) } \\
\text { Change in NAS: }-1.3 \\
\text { vs. }-0.8, P=0.24 \\
\text { Change in fibrosis stage: }-0.2 \\
\text { vs. } 0.2, P=0.11 \\
\text { Patients with improvement } \\
\text { in fibrosis: } 26 \% \text { vs. } 14 \% \text {, } \\
P=0.46 \\
\text { Patient with worsening } \\
\text { fibrosis: } 9 \% \text { vs. } 36 \%, \\
P=0.04 \\
\text { Change in ALT: }-26.6 \\
\text { vs. }-10.2, P=0.16 \\
\text { Change in AST: }-27 \\
\text { vs. }+9 \mathrm{IU} / \mathrm{L} ; P=0.025\end{array}$ & $\begin{array}{l}\text { WAE: } 8 \% \text { vs. } 4 \% \\
(P=0.56) \\
\text { SAE: } 8 \% \text { vs. } 8 \% \\
\text { GI disorders: } 81 \% \text { vs. } \\
65 \%(P=0.27)\end{array}$ \\
\hline Shao, 2014 [8] China (fair) & $\begin{array}{l}\text { Patients with type } 2 \\
\text { diabetes, obesity, NAFLD, } \\
\text { and elevated liver enzymes } \\
\text { with normal renal function } \\
\text { Age: } 43 \\
\text { Sex: } 48 \% \text { male } \\
\text { Ethnicity: Chinese } \\
\text { Mild NAFLD: } 40 \% \\
\text { Moderate NAFLD: } 42 \% \\
\text { Severe: } 18 \% \\
\text { BMI: } 30 \\
\text { HbA1c: } 7.64 \%\end{array}$ & $\begin{array}{l}\text { A. exenatide + glargine (30) } \\
\text { B. Intensive insulin: Insulin } \\
\text { aspart + insulin glargine (30) } \\
\text { Duration: } 12 \text { weeks }\end{array}$ & $\begin{array}{l}\text { Reversal rate of NAFLD } \\
\text { based on ultrasound: } \\
\text { A vs. B: } 93 \% \text { vs. } 67 \%, \\
P<0.01 \\
\text { Differences in weight } \\
\text { change post minus } \\
\text { pretreatment: } \\
\text { A vs. B: }-7.77 \text { kg vs. } 3.27 \text {, } \\
P<0.001 \\
\text { No difference between } \\
\text { groups in change in HbA1c: } \\
\text { A vs. B: }-1.42 \% \text { vs. }-1.31 \% \text {, } \\
P>0.05\end{array}$ & NR \\
\hline
\end{tabular}

\section{Exenatide}

A trial of exenatide $(N=132)$ conducted in China enrolled patients with NAFLD and diabetes and reported efficacy outcomes only [8]. Twelve weeks of exenatide treatment ( $5 \mathrm{mg}$ twice daily for 4 weeks then $10 \mathrm{mg}$ twice daily for 8 weeks) was associated with greater reversal of liver fat assessed by ultrasound than intensive insulin therapy $(93 \%$ vs. $67 \%, P<0.01)[8]$. At the conclusion of 
Table 3 Studies of metformin to treat nonalcoholic fatty liver disease

\begin{tabular}{|c|c|c|c|c|}
\hline \multirow{2}{*}{$\begin{array}{l}\text { Author, year country trial } \\
\text { name (quality rating) }\end{array}$} & \multirow[t]{2}{*}{ Population demographics } & \multirow{2}{*}{$\begin{array}{l}\text { Interventions (group sizes) } \\
\text { duration }\end{array}$} & \multirow{2}{*}{$\begin{array}{l}\text { Efficacy/effectiveness outcomes } \\
\text { A vs. B }\end{array}$} & \multirow{2}{*}{$\begin{array}{l}\text { Harms } \\
\text { A vs. B }\end{array}$} \\
\hline & & & & \\
\hline $\begin{array}{l}\text { Anushiravani, } 2019 \\
\text { [22] Iran (good) }\end{array}$ & $\begin{array}{l}\text { Adults with probable } \\
\text { NAFLD with or without } \\
\text { elevated } \\
\text { ALT/AST } \\
\text { Age: } 47 \text { y } \\
\text { \% female: } 49 \\
\text { Ethnicity: NR } \\
\text { BMl, } \mathrm{kg} / \mathrm{m}^{2}: 25.1 \text { vs. } 26.1\end{array}$ & $\begin{array}{l}\text { A. Metformin } 500 \mathrm{mg} / \mathrm{d} \\
(n=30) \\
\text { B. Placebo }(n=30) \\
\text { Duration: } 3 \text { months }\end{array}$ & $\begin{array}{l}\text { Changes from baseline } \\
\text { ( } P \text { value), between-groups } \\
P \text { value: } \\
\text { BMl: }-0.6 \text { vs. }-0.7 \mathrm{~kg} / \mathrm{m}^{2} ; P=\mathrm{NS} \\
\text { ALT: }-10.1 \text { vs. }-0.6 ; P<0.001 \\
\text { AST: }-6.4 \text { vs. }-0.9 ; P<0.001\end{array}$ & None \\
\hline $\begin{array}{l}\text { Haukeland, } 2009 \\
\text { [15] Norway (fair) }\end{array}$ & $\begin{array}{l}\text { Adults with biopsy- } \\
\text { confirmed NAFLD } \\
\text { Age: } 47.4 \text { y } \\
\% \text { female: } 27.2 \\
\text { Ethnicity, \% white: } 86.4 \% \\
\text { BMl, } \mathrm{kg} / \mathrm{m}^{2}: 30.8 \\
\text { Diabetes: } 27.3 \%\end{array}$ & $\begin{array}{l}\text { A: Metformin } 2500 \mathrm{mg} / \mathrm{d} \\
(3000 \mathrm{mg} \text { if weight }>90 \mathrm{~kg}) \\
(n=24) \\
\text { B: Placebo }(n=24) \\
\text { Duration: } 6 \text { months }\end{array}$ & $\begin{array}{l}\text { Percentage with improvement } \\
(P \text { value change from baseline); } \\
\text { between-groups } P \text { value: } \\
\text { Steatosis: } 25 \%(P=0.10) \text { vs. } 38 \% \\
(P=0.03) ; P=0.052 \\
\text { Fibrosis: } 5 \%(P=1.00) \text { vs. } 17 \% \\
(P=0.17) ; P=0.36 \\
\text { NAFLD activity score: } 20 \% \\
(P=0.23) \text { vs. } 50 \%(P=0.12) ; \\
P=0.06 \\
\text { Changes from baseline ( } P \text { value); } \\
\text { between-groups } P \text { value: } \\
\text { Weight, kg: }-4.3(P<0.001) \text { vs. } 0.3 \\
(P=0.45) ; P<0.001 \\
\text { BMl: } 1.3(P<0.001) \text { vs. } 0.1 \\
(P=0.59) ; P<0.001\end{array}$ & $\begin{array}{l}\text { Serious AEs: NR } \\
\text { Withdrawal due to } \\
\text { AEs: } 2 / 24(8.3 \%) \text { vs. } \\
\text { 0/24 (0\%) }\end{array}$ \\
\hline $\begin{array}{l}\text { Omer, } 2010[20] \\
\text { Turkey (fair) }\end{array}$ & $\begin{array}{l}\text { Adults with type } 2 \\
\text { diabetes or impaired } \\
\text { glucose tolerance and } \\
\text { biopsy-confirmed } \\
\text { NAFLD } \\
\text { Age: } 48.9 \text { y } \\
\% \text { female: } 45.3 \\
\text { Ethnicity: NR } \\
\text { BMl, } \mathrm{kg} / \mathrm{m}^{2}: 30.6 \\
\text { Diabetes: NR }\end{array}$ & $\begin{array}{l}\text { A: Metformin } 1700 \mathrm{mg} / \mathrm{d} \\
\text { + rosiglitazone } 4 \mathrm{mg} / \mathrm{d} \\
(n=22) \\
\text { B: Metformin } 1700 \mathrm{mg} / \mathrm{d} \\
(n=22) \\
\text { C: Rosiglitazone } 4 \mathrm{mg} / \mathrm{d} \\
(n=20) \\
\text { Duration: } 12 \text { months }\end{array}$ & $\begin{array}{l}\text { Changes from baseline ( } P \text { value): } \\
\text { NAFLD score }(n=10-13):-3.9 \\
(P=0.026) \text { vs. } 0.7(P=0.726) \\
\text { vs. }-2.6(P=0.012) \\
\text { AST: }-15.4(P=0.01) \text { vs. }-13.0 \\
(P=\text { NS) vs. }-13.2(P=0.005) \\
\text { ALT: }-22.7(P=0.017) \text { vs. }-16.7 \\
(P=\text { NS) vs. }-36.2(P<0.0001) \\
\text { BMI: }-1.3(P=0.006) \text { vs. }-3.2 \\
(P=0.002) \text { vs. }-0.3(P=\text { NS })\end{array}$ & $\begin{array}{l}\text { Serious AEs: NR } \\
\text { Withdrawal due to } \\
\text { AEs: Not adequately } \\
\text { reported }\end{array}$ \\
\hline $\begin{array}{l}\text { Rana, } 2016 \text { [10] } \\
\text { India (fair) }\end{array}$ & $\begin{array}{l}\text { Patients with ultrasound } \\
\text { diagnosed NAFLD without } \\
\text { history of use of insulin } \\
\text { sensitizers or hypolipidemic } \\
\text { drug use } \\
\text { Age: NR } \\
\text { Sex: NR } \\
\text { Ethnicity: Indian } \\
\text { Liver status: AST } 55.14 \mathrm{IU} / \mathrm{mL} \text {; } \\
\text { ALT } 64.30 \text { (AST and ALT were } \\
\text { different at baseline between } \\
\text { treatment groups) } \\
\text { BMl: } 27.95\end{array}$ & $\begin{array}{l}\text { A. Metformin (31) } \\
\text { B. Rosuvastatin (34) } \\
\text { C. Pioglitazone (33) } \\
\text { Duration: } 24 \text { weeks }\end{array}$ & $\begin{array}{l}\text { Change in ultrasound score } \\
\text { (fatty liver) at } 24 \text { weeks: our analysis } \\
\text { A vs. B: } 0.065 \text { vs. }-1.265(P<0.001) \\
\text { A vs. C: } 0.065 \text { vs. }-0.697(P<0.001) \\
\text { Weight change at } 24 \text { weeks: our } \\
\text { analysis } \\
\text { A vs. B: }-4.76 \text { vs. }-4.25(P=0.13) \\
\text { A vs. C: }-4.76 \text { vs. } 0.03(P<0.001) \\
\text { AST change at } 24 \text { weeks: our analysis } \\
\text { A vs. B: }-14.07 \text { vs. } 8.35(P<0.001) \\
\text { A vs. C: }-14.07 \text { vs. }-23.73(P=0.04) \\
\text { ALT change at } 24 \text { weeks: our analysis } \\
\text { A vs. B: }-15.55 \text { vs. } 8.06(P<0.001) \\
\text { A vs. C: }-15.55 \text { vs. }-24.67(P=0.13)\end{array}$ & NR \\
\hline $\begin{array}{l}\text { Razavizade, } 2013 \text { [14] } \\
\text { Iran (fair) }\end{array}$ & $\begin{array}{l}\text { Adults with NAFLD } \\
\text { assessed via } \\
\text { ultrasonography } \\
\text { and predictive formula } \\
\text { Age: } 35.3 \text { y } \\
\% \text { female: } 15 \\
\text { Ethnicity: NR } \\
\text { BMI, } \mathrm{kg} / \mathrm{m}^{2}: 27.7 \\
\text { Diabetes: } 7.5 \%\end{array}$ & $\begin{array}{l}\text { A: Metformin } 1000 \mathrm{mg} / \mathrm{d} \\
(n=40) \\
\text { B: Pioglitazone } 30 \mathrm{mg} / \mathrm{d} \\
\text { ( } n=40) \\
\text { Duration: } 4 \text { months }\end{array}$ & $\begin{array}{l}\text { Changes from baseline ( } P \text { value), } \\
\text { between-groups } P \text { value: } \\
\text { Liver fat fraction: }-2.53(P<0.01) \\
\text { Vs. }-3.23(P<0.01), P=0.48 \\
\text { AST: }-10.83(P<0.01) \text { vs. }-13.75 \\
(P<0.01), P=0.56 \\
\text { ALT: }-21.75(P<0.01) \text { vs. }-37.53 \\
(P<0.01), P=0.07 \\
\text { Weight, } \mathrm{kg}:-2.73(P<0.01) \\
\text { vs. }-1.18(P=0.04), P=0.05\end{array}$ & $\begin{array}{l}\text { Serious AEs: NR } \\
\text { Withdrawal due to } \\
\text { AEs: None. }\end{array}$ \\
\hline
\end{tabular}

therapy, 40 to $43 \%$ of patients no longer had a fatty liver, and in the exenatide group, 0 out of 6 patients $(0 \%)$ still had severe disease compared with 3 out of 5 patients $(60 \%)$ in the insulin group ( $P=0.03$, our analysis). Body weight was also significantly reduced with exenatide compared with intensive insulin $(-7.77 \mathrm{~kg}$ vs. $+3.27 \mathrm{~kg}$, $P<0.001)$ but there was no differential treatment effect on HbA1c (- $1.42 \%$ vs. $-1.31 \%, P>0.05)$. 
Table 4 Studies of DPP-4 inhibitors to treat nonalcoholic fatty liver disease

\begin{tabular}{|c|c|c|c|c|}
\hline $\begin{array}{l}\text { Author, year country trial } \\
\text { name (quality rating) }\end{array}$ & Population demographics & Interventions (group sizes) duration & $\begin{array}{l}\text { Efficacy/effectiveness outcomes } \\
\text { A vs. B }\end{array}$ & $\begin{array}{l}\text { Harms } \\
\text { A vs. B }\end{array}$ \\
\hline $\begin{array}{l}\text { Deng, } 2017 \text { [7] } \\
\text { China (good) }\end{array}$ & $\begin{array}{l}\text { Patients with type } 2 \text { diabetes } \\
\text { for less than } 2 \text { years without } \\
\text { complications and fatty liver } \\
\text { diagnosed by ultrasound } \\
\text { Age: } 64 \\
\text { Sex: } 75 \% \text { male } \\
\text { Ethnicity: } \\
\text { Liver status: ALT } 35 \mathrm{IU} / \mathrm{mL} \text {; AST: } \\
32 \mathrm{IU} / \mathrm{mL} \\
\text { BMl: } 24 \\
\text { Diabetes: HbA1c } 7.4 \%\end{array}$ & $\begin{array}{l}\text { A. Sitagliptin } 50 \text { to } 100 \mathrm{mg}(36) \\
\text { B. Diet and exercise } \\
\text { Duration: } 52 \text { weeks }\end{array}$ & $\begin{array}{l}\text { No difference in change in AST } \\
(P=0.99) \text { or ALT }(P=0.97) \text { between } \\
\text { treatment with sitagliptin vs. diet and } \\
\text { exercise } \\
\text { Greater decrease in HbA1c with } \\
\text { sitagliptin }(-0.81) \text { vs. diet and } \\
\text { exercise }(-0.25), P<0.01 \text { at } \\
52 \text { weeks (also at } 13,26 \text {, and } 39 \text { weeks) }\end{array}$ & NR \\
\hline
\end{tabular}

\section{Sitagliptin}

A small RCT conducted in China found no difference between sitagliptin 50 to $100 \mathrm{mg}$ compared with diet and exercise on the liver function tests AST and ALT after 1 year of treatment, although sitagliptin treatment was associated with greater reduction in HbA1c $(-0.81$ vs. $-0.25, P<0.01)[7]$.

\section{Pioglitazone}

Two RCTs enrolled patients with histologically confirmed NASH and prediabetes or diabetes as determined by an abnormal glucose tolerance test $[18,19]$. In the first trial, all 101 patients were advised to follow a hypocaloric diet $(500 \mathrm{kcal} / \mathrm{d}$ deficit) and were then randomized to pioglitazone $45 \mathrm{mg}$ or placebo for 18 months (some patients were also taking metformin, a sulfonylurea, and/or insulin) [18]. A greater than or equal to 2point reduction in Nonalcoholic fatty liver disease Activity Score (NAS) without worsening of fibrosis was the primary outcome and favored pioglitazone (58\% vs. $18 \%$, RR 3.28 , 95\% CI 1.74 to 6.22 , our analysis). Resolution of NASH based on liver histology also favored pioglitazone (52\% vs. 20\%, RR 2.65, 95\% CI 1.43 to 4.91 , our analyses). HbA1c improved slightly with pioglitazone compared with placebo $(-0.6 \%, P=0.009)$; however, treatment with pioglitazone was also associated with significant gain in weight $(2.5 \mathrm{~kg}, P=0.02)$.

In the second trial, 55 patients were counseled to follow a hypocaloric diet and then randomized to treatment with pioglitazone $45 \mathrm{mg}$ or placebo for 6 months [19]. Pioglitazone treatment versus placebo was associated with greater improvements in HbA1c $(-0.7 \%$ vs. $-0.1 \%, P=0.008)$, AST ( -19 vs. $-9 \mathrm{IU} / \mathrm{L}, P=0.04)$, and ALT ( -39 vs. -21 IU/L, $P<0.001)$. The proportion of participants who experienced improvement in hepatic fat was greater in the pioglitazone group ( $65 \%$ vs. $38 \%, P=0.003$ ), while there were no differences between treatments in fibrosis score. Pioglitazone treatment was also associated with weight gain compared with placebo $(+2.5 \mathrm{~kg}$ vs. $-0.5 \mathrm{~kg}, P=$ $0.003)$. Study withdrawals due to adverse events were few and not different between groups.

\section{Rosiglitazone and metformin}

One trial randomized 64 patients with NAFLD to treatment with rosiglitazone $4 \mathrm{mg} /$ day, metformin $1700 \mathrm{mg} /$ day, or combination therapy for 12 months [20]. All patients also had impaired glucose metabolism (type 2 DM or impaired glucose tolerance) and elevated liver transaminases and were on a diet and exercise program for 12 weeks prior to the start of the trial. Baseline insulin levels were significantly different between groups (10.1 $\mathrm{mg} / \mathrm{dL}$ in the rosiglitazone group, $14.9 \mathrm{mg} / \mathrm{dL}$ in the metformin group, $16.6 \mathrm{mg} / \mathrm{dL}$ in the combined therapy group, $P=0.04$ ) but not significantly different between groups in other baseline characteristics. The trial reported decreased BMI from baseline for 12 months for metformin ( 30.8 to $\left.27.6 \mathrm{~kg} / \mathrm{m}^{2}, P=0.002\right)$ and for rosiglitazone plus metformin ( 32.5 to $\left.31.2 \mathrm{~kg} / \mathrm{m}^{2}, P=0.006\right)$. However, we believe that the values for rosiglitazone plus metformin to be in error as the correlation between baseline and 12 months would have to be greater than 0.96, which is extremely high and not likely correct. Postprandial glucose was decreased in all groups. Posttreatment liver biopsy was performed on $55 \%$ of patients and NAFLD activity score favored treatment with rosiglitazone $(P=0.01)$ and combination therapy $(P=0.03)$. Three individuals left the study, and two due to adverse events (one patient could not tolerate metformin and one stopped treatment due to hypertriglyceridemia).

\section{Patients with NAFLD (without diabetes)}

Nine trials enrolled NAFLD patients without diabetes and included metformin [10, 15, 22, 23],\} pioglitazone $[10-13,22]$,$\} liraglutide [9], rosiglitazone [17], and rosi-$ glitazone with metformin [16].

\section{Liraglutide}

A small trial $(N=52)$ of liraglutide compared with placebo conducted in the UK in adults with NASH (LEAN trial) allowed enrollment of patients with diabetes if the diabetes was well-controlled and stable (33\% had diabetes) [9]. More patients taking liraglutide had a resolution of NASH at 48 weeks than with placebo (39\% vs. 
9\%, RR 4.3, 95\% CI 1.0 to 17.7). There was no difference in change in NAFLD activity score $(-1.3$ vs. $-0.8, P=$ $0.24)$, fibrosis score $(-0.2$ vs. $0.2, P=0.11)$, ALT (26.6 vs. $10.2 \mathrm{IU} / \mathrm{L}, P=0.16$ ), or AST (- 15.8 vs. -8.6 $\mathrm{IU} / \mathrm{L}, P=0.29)$. However, significantly more patients on placebo had worsening fibrosis (36\% vs. 9\%, RR 0.2 , 95\% CI 0.1 to $1.0, P=0.04)$. There was greater weight loss with liraglutide $(-5.3 \mathrm{~kg}$ vs. $-0.6 \mathrm{~kg}, \mathrm{RR}-4.39 \mathrm{~kg}$, $95 \% \mathrm{CI}-7.19$ to $-1.59 \mathrm{~kg}$ ) and greater improvement in HbA1c (- $0.53 \%$ vs. $0 \%$, RR $-0.48 \%, 95 \%$ CI -0.91 to $0.05 \%)$. There were no differences between treatments in study withdrawals due to adverse events, risk of serious adverse events, or risk of gastrointestinal disorders.

\section{Metformin}

One small trial $(N=48)$ conducted in Norway randomized patients with NAFLD to treatment with metformin $2500 \mathrm{mg}$ (3000 mg if body weight $>90 \mathrm{~kg}$ ) or placebo for 6 months [15]. Twenty-seven percent of patients had diabetes. There was no difference between groups at end of treatment in steatosis, NAFLD activity score, or fibrosis. However, weight loss was greater with metformin than placebo $(-4.4 \mathrm{~kg}$ vs. $+0.3 \mathrm{~kg}, P<0.001)$ as was reduction in HbA1c $(-0.2 \%$ to $+0.1 \%, P=0.001)$ [23].

A second good-quality trial assessed 3 months of metformin $(500 \mathrm{mg} /$ day) use among Iranian adults with probable NAFLD by liver sonography [22]. Compared with placebo $(n=30)$, there was no difference among patients receiving metformin $(n=30)$ in change in BMI (- 0.6 vs. $-0.7 ; P=0.91$ ), though ALT (- 10.1 vs. -0.6 IU/L) and AST (- 6.4 vs. - 0.9 IU/L) were significantly reduced among those taking metformin. No adverse events were reported.

\section{Pioglitazone}

Three trials randomized patients without diabetes to pioglitazone or placebo [12, 13, 22]. In the first RCT, 163 patients with NASH were randomized to $30-\mathrm{mg}$ pioglitazone or to placebo for 96 weeks [13]. Most patients (87\%) underwent end-of-study biopsy. More patients treated with pioglitazone compared with placebo experienced improvement in liver histology (34\% vs. $19 \%, P=$ $0.04)$, steatosis $(69 \%$ vs. $31 \%, P<0.001)$, NAFLD score ( -1.9 vs. $10.5, P<0.001)$, and resolution of NASH $(47 \%$ vs. $21 \%, P=0.001)$. Liver function tests, fasting serum glucose, and insulin resistance were also significantly improved with pioglitazone versus placebo. However, weight gain was increased with pioglitazone compared with placebo $(+4.7 \mathrm{~kg}$ vs. $+0.7 \mathrm{~kg}, P<0.001)$. There was no difference between groups in change in liver fibrosis. Twelve patients experienced serious adverse events, with fewer events in the pioglitazone group $(2.5 \%$ vs. $12 \%$, RR $0.21,95 \%$ CI 0.05 to 0.92 ).
In the second RCT, 74 patients with NASH were randomized to $30 \mathrm{mg}$ of pioglitazone or placebo for 12 months [12]. All received diet and exercise counseling that was reinforced each visit. Sixty-one participants $(82 \%)$ had liver biopsy at the end of treatment. A reduction in liver fat was seen with pioglitazone and placebo from baseline with no difference between groups. Pioglitazone was associated with a reduction in liver fibrosis compared with placebo $(P=0.05)$. Pioglitazone was also associated with greater improvement in HbA1c compared with placebo $(-0.2 \%$ vs. $+0.1 \%, P=0.01)$, as well as greater improvement in ALT (- 37.1 vs. - 6.9 IU/L, $P=0.009$ ). However, as above, pioglitazone treatment was associated with increase in body weight versus placebo $(+2.6 \mathrm{~kg}$ vs. $3.5 \mathrm{~kg}, P=0.02)$. Withdrawals due to adverse events (18\%) were similar between groups.

A third good-quality trial compared pioglitazone (15 $\mathrm{mg} /$ day) with placebo ( $n=30$ in each group) among Iranian adults with probable NAFLD by liver sonography [22]. While BMI was not different between groups $(-0.6$ vs. $\left.-0.7 \mathrm{~kg} / \mathrm{m}^{2} ; P=0.46\right)$, ALT $(-8.6$ vs. $-0.6 \mathrm{IU} / \mathrm{L} ; P<$ $0.001)$ and AST $(-6.7$ vs. $-0.9 \mathrm{IU} / \mathrm{L} ; P<0.001)$ were significantly reduced among those receiving pioglitazone. No adverse events were reported.

\section{Metformin or pioglitazone}

In a randomized trial conducted in India, 98 patients were allocated to metformin, rosuvastatin, or pioglitazone [10]. At 24 weeks, change in ultrasound fatty liver score significantly favored rosuvastatin and pioglitazone over metformin (- 1.27 vs. -0.70 vs. $+0.07, P<0.001)$. Change in BMI favored metformin and rosuvastatin over pioglitazone (- 1.75 vs. -1.54 vs. $\left.-0.15 \mathrm{~kg} / \mathrm{m}^{2}, P<0.001\right)$, whereas change in AST favored pioglitazone and metformin over rosuvastatin $(-23.73 \mathrm{IU} / \mathrm{L}$ vs. $-14.07 \mathrm{IU} / \mathrm{L}$ vs. + 8.06, $P=0.012)$. Adverse events were not reported.

A second trial that randomized 80 NAFLD patients to treatment with metformin $1000 \mathrm{mg}$ or pioglitazone 30 mg for 4 months was conducted in Iran [14]. Eight percent of patients had diabetes. Both metformin and pioglitazone decreased body weight, liver function tests, fasting plasma glucose, and liver fat from baseline with no differences between treatments. No participant left the study due to adverse events or required a medication dose adjustment.

\section{Pioglitazone or pentoxifylline}

One randomized trial $(N=60)$ conducted in India compared 6-month treatment with pioglitazone $30 \mathrm{mg}$ to treatment with pentoxifylline $1200 \mathrm{mg}$ in patients with biopsy-proven NASH [11]. All patients received diet and exercise counseling. AST and ALT were both improved from baseline with pioglitazone $(P=0.003$, both comparisons) but were not different from improvements with 
pentoxifylline. Improvements in liver fat and fasting blood sugar were also improved from baseline with pioglitazone ( $P=0.005 ; P=0.02$, respectively) but were also not significantly different from improvement with pentoxifylline. Although patients gained weight with pioglitazone, the increase $(2 \mathrm{~kg})$ was not statistically significant $(P=0.31)$. No participant left the study due to adverse events.

\section{Rosiglitazone}

One RCT randomized 63 patients with NASH to rosiglitazone $(4 \mathrm{mg} /$ day for 1 month then $8 \mathrm{mg} /$ day for 11 months) or placebo for 12 months [17] and found a reduction in liver fat with rosiglitazone ( $47 \%$ vs. $16 \%, P=$ 0.01 ), along with increased normalization of transaminases (38\% vs. $7 \%, P=0.005)$ but an increase in weight $(+1.5 \mathrm{~kg}$ vs. $-1.0 \mathrm{~kg}, P<0.01)$. There were no differences between groups in NAFLD Activity Score or HbA1c levels between treatments, although surrogate markers of insulin sensitivity were improved with rosiglitazone. Three patients treated with rosiglitazone experienced painful, swollen legs requiring dose adjustment, or discontinuation of treatment.

\section{Rosiglitazone and metformin}

One RCT randomized patients with NASH to $4 \mathrm{mg}$ of rosiglitazone or to $4 \mathrm{mg}$ of rosiglitazone plus $1000 \mathrm{mg}$ of metformin or to $4 \mathrm{mg}$ of rosiglitazone plus $50 \mathrm{mg}$ of losartan for 48 weeks [16]. Seventeen percent of participants screened positive for diabetes (HbA1c > 6.5\%). The baseline NAFLD activity score was different between groups (highest in the rosiglitazone-alone group at 5.1 and lowest in the rosiglitazone plus metformin group at $4.1, P=0.014$ ). Liver fat and fibrosis stages were similar between groups. Post-treatment liver biopsies showed no differences between groups on changes in NAFLD score, steatosis, fibrosis, or resolution of NASH (46\% of 26 patients treated with rosiglitazone versus 36\% of 28 patients treated with rosiglitazone and metformin). Liver function tests were improved in all treatment groups as was fasting serum glucose and insulin levels. The addition of metformin did not significantly help with weight gain versus treatment with rosiglitazone alone ($1.2 \mathrm{~kg}$ vs. $+0.9 \mathrm{~kg}, P=0.051)$. Twelve patients, at the recommendation of their physicians, terminated the study due to 12 different adverse events, some likely unrelated to treatment (e.g., terminal cancer).

\section{Discussion}

Management of NAFLD involves treating the liver disease itself as well as associated metabolic comorbidities, including diabetes, obesity, and hyperlipidemia. A recent clinical practice guideline from the American Association for the Study of Liver Diseases (AASLD) [24] recommended that pharmacologic treatment aimed primarily at improving liver disease be limited to patients with biopsy-proven NASH and fibrosis, since patients without fibrosis generally have a favorable prognosis. As such, first-line treatment for most patients should focus on lifestyle intervention or pharmacologic treatment targeting diagnosed diabetes, obesity, or dyslipidemia. The AASLD guidelines recommend weight loss via hypocaloric diet and increased exercise, with a target of at least 7-10\% weight loss to improve the majority of the histopathological features of NAFLD. The group recommends against metformin or GLP-1 agonists and recommends pioglitazone (a thiazolidinedione) in patients with biopsy-proven NASH, regardless of diabetes status, but recommends against using pioglitazone in NAFLD patients without fibrosis.

Consistent with the AASLD recommendation against metformin use in NAFLD patients, studies of metformin found no difference from placebo in steatosis, fibrosis, NAFLD activity score, or resolution of NASH. While weight and glucose control were improved with metformin, treatment with metformin did not substantially impact liver disease.

Studies of pioglitazone in NASH patients found benefits in liver function, liver fat, and NASH resolution, though significant increases in weight may be cause for concern. A recent systematic review and network metaanalysis by Sridharan et al. was consistent with our findings [25]: pioglitazone was found to be associated with better response than standard care (odds ratio 3.8, 95\% $\mathrm{CI}, 2.0$ to 7.4). Evidence for other thiazolinediones was more limited and had somewhat mixed results, but findings were generally consistent with those for pioglitazone: liver fat and function and glucose measures improved, but weight also increased.

While the AASLD guidelines recommend against using GLP-1 agonists in NASH patients, we found some evidence that liraglutide improves liver fat, liver function, and $\mathrm{HbA} 1 \mathrm{c}$ and is effective at resolving $\mathrm{NASH}$ and reducing weight. Exenatide performed less well but also resulted in significant reductions in liver fat and weight.

The strengths of our study include the use of systematic review processes to identify all relevant studies that meet pre-defined inclusion criteria, assessment of the internal validity (i.e., quality) of included studies, and overall evaluation of the strength of evidence using an established approach. Limitations of the present review include restriction to English-language publications and restriction to randomized trials, which may have limited generalizability to real-world populations. Larger studies with longer follow-up are needed to better quantify the long-term benefits and harms of diabetes medications to treat NAFLD, since the disease state itself, as well as many of the common metabolic conditions associated with NAFLD, are chronic conditions with long natural 
histories. Additionally, longer and larger studies may provide further information on clinical health outcomes and uncommon adverse effects.

\section{Conclusions}

Consistent with existing clinical practice guidelines, which recommend lifestyle intervention and treatment for comorbidities related to fatty liver disease as firstline treatment, trial evidence supports the efficacy of some diabetes drugs (especially pioglitazone) in patients with NAFLD or NASH, though weight gain with some diabetes drugs may warrant caution. Larger trials are needed to better characterize the efficacy and harms of diabetes pharmacotherapy in these patients.

\section{Supplementary information}

Supplementary information accompanies this paper at https://doi.org/10. 1186/s13643-019-1200-8.

Additional file 1. Search strategies

\section{Abbreviations}

AASLD: American Association for the Study of Liver Diseases; AHRQ: Agency for Healthcare Research \& Quality; ALT: Alanine aminotransferase; AST: Aspartate aminotransferase; Cl: Confidence interval; DERP: Drug Effectiveness Review Project; FDA: Food and Drug Administration; HbA1c: Glycated hemoglobin; IPD: Individual patient data; NAFLD: Nonalcoholic fatty liver disease; NASH: Nonalcoholic steatohepatitis; RCT: Randomized controlled trial; RR: Relative risk

\section{Acknowledgements}

Marian McDonagh, PharmD, lead the Drug Effectiveness Review Project and contributed to the report on which this manuscript is based. Sam Liebow provided logistical and administrative support.

\section{Authors' contributions}

IB drafted the manuscript, which was based on a report conducted and drafted jointly by IB and SS. All authors read and approved the final manuscript.

\section{Funding}

Research was funded by the Drug Effectiveness Review Project; participating members contributed to determining the scope of the review but were not involved in data collection, analysis, interpretation, or writing of the manuscript.

\section{Availability of data and materials}

All data generated or analyzed during this study are included in this published article (and its supplementary information files).

\section{Ethics approval and consent to participate}

Not applicable.

\section{Consent for publication}

Not applicable.

\section{Competing interests}

The authors declare that they have no competing interests.

Received: 4 April 2019 Accepted: 15 October 2019

Published online: 29 November 2019

\section{References}

1. National Guideline C. National Institute for Health and Care Excellence: Guidance. Non-alcoholic fatty liver disease: assessment and management.
London: National Institute for Health and Care Excellence (UK) Copyright (c) National Institute for Health and Care Excellence 2016: 2016.

2. Younossi ZM, Koenig AB, Abdelatif D, Fazel Y, Henry L, Wymer M. Global epidemiology of nonalcoholic fatty liver disease-meta-analytic assessment of prevalence, incidence, and outcomes. Hepatology. 2016;64(1):73-84.

3. Pais R, Barritt AS, Calmus Y, Scatton O, Runge T, Lebray P, et al. NAFLD and liver transplantation: current burden and expected challenges. Journal of hepatology. 2016;65(6):1245-57.

4. Cernea S, Cahn A, Raz I. Pharmacological management of nonalcoholic fatty liver disease in type 2 diabetes. Expert review of clinical pharmacology. 2017;10(5):535-47.

5. McDonagh MS, Jonas DE, Gartlehner G, Little A, Peterson K, Carson S, et al. Methods for the drug effectiveness review project. BMC Medical Research Methodology. 2012;12:140

6. Berkman N, Lohr K, Ansari M, McDonagh M, Balk E, Whitlock E. AHRO Methods for effective health care: grading the strength of a body of evidence when assessing health care interventions for the effective health care program of the agency for healthcare research and quality: an update. Methods guide for effectiveness and comparative effectiveness reviews Rockville (MD): agency for healthcare research and quality (US). 2008.

7. Deng XL, Ma R, Zhu HX, Zhu J. Short article: a randomized-controlled study of sitagliptin for treating diabetes mellitus complicated by nonalcoholic fatty liver disease. European Journal of Gastroenterology \& Hepatology. 2017;29(3):297-301.

8. Shao N, Kuang HY, Hao M, Gao XY, Lin WJ, Zou W. Benefits of exenatide on obesity and non-alcoholic fatty liver disease with elevated liver enzymes in patients with type 2 diabetes. Diabetes/Metabolism Research Reviews. 2014; 30(6):521-9.

9. Armstrong MJ, Gaunt P, Aithal GP, Barton D, Hull D, Parker R, et al. Liraglutide safety and efficacy in patients with non-alcoholic steatohepatitis (LEAN): a multicentre, double-blind, randomised, placebo-controlled phase 2 study. Lancet. 2016:387(10019):679-90.

10. Rana H, Yadav SS, Reddy HD, Singhal S, Singh DK, Usman K. Comparative effect of insulin sensitizers and statin on metabolic profile and ultrasonographical score in non alcoholic fatty liver disease. Journal of Clinical and Diagnostic Research. 2016:10(8):OC19-23.

11. Sharma BC, Kumar A, Garg V, Reddy RS, Sakhuja P, Sarin SK. A randomized controlled trial comparing efficacy of pentoxifylline and pioglitazone on metabolic factors and liver histology in patients with non-alcoholic steatohepatitis. Journal of Clinical and Experimental Hepatology. 2012;2(4): 333-7.

12. Aithal GP, Thomas JA, Kaye PV, Lawson A, Ryder SD, Spendlove I, et al. Randomized, placebo-controlled trial of pioglitazone in nondiabetic subjects with nonalcoholic steatohepatitis. Gastroenterology. 2008;135(4):1176-84.

13. Sanyal AJ, Chalasani N, Kowdley KV, McCullough A, Diehl AM, Bass NM, et al. Pioglitazone, vitamin $\mathrm{E}$, or placebo for nonalcoholic steatohepatitis. New England Journal of Medicine. 2010:362(18):1675-85.

14. Razavizade M, Jamali R, Arj A, Matini SM, Moraveji A, Taherkhani E. The effect of pioglitazone and metformin on liver function tests, insulin resistance, and liver fat content in nonalcoholic fatty liver disease: a randomized double blinded clinical trial. Hepatitis Monthly. 2013:13:5.

15. Haukeland JW, Konopski Z, Eggesbo HB, von Volkmann HL, Raschpichler G, Bjoro $\mathrm{K}$, et al. Metformin in patients with non-alcoholic fatty liver disease: a randomized, controlled trial. Scandinavian Journal of Gastroenterology. 2009:44(7):853-60

16. Torres DM, Jones FJ, Shaw JC, Williams CD, Ward JA, Harrison SA. Rosiglitazone versus rosiglitazone and metformin versus rosiglitazone and losartan in the treatment of nonalcoholic steatohepatitis in humans: a 12month randomized, prospective, open- label trial. Hepatology. 2011;54(5): $1631-9$

17. Ratziu V, Giral P, Jacqueminet S, Charlotte F, Hartemann-Heurtier A, Serfaty $L$, et al. Rosiglitazone for nonalcoholic steatohepatitis: one-year results of the randomized placebo-controlled Fatty Liver Improvement with Rosiglitazone Therapy (FLIRT) Trial. Gastroenterology. 2008;135(1):100-10.

18. Cusi K, Orsak B, Bril F, Lomonaco R, Hecht J, Ortiz-Lopez C, et al. Long-term pioglitazone treatment for patients with nonalcoholic steatohepatitis and prediabetes or type 2 diabetes mellitus: a randomized trial. Annals of Internal Medicine. 2016;165(5):305-15.

19. Belfort R, Harrison SA, Brown K, Darland C, Finch J, Hardies J, et al. A placebo-controlled trial of pioglitazone in subjects with nonalcoholic steatohepatitis. New England Journal of Medicine. 2006;355(22):2297-307. 
20. Omer Z, Cetinkalp S, Akyildiz M, Yilmaz F, Batur Y, Yilmaz C, et al. Efficacy of insulin-sensitizing agents in nonalcoholic fatty liver disease. European Journal of Gastroenterology \& Hepatology. 2010;22(1):18-23.

21. Armstrong MJ, Houlihan DD, Rowe IA, Clausen WH, Elbrond B, Gough SC, et al. Safety and efficacy of liraglutide in patients with type 2 diabetes and elevated liver enzymes: individual patient data meta-analysis of the LEAD program. Alimentary Pharmacology \& Therapeutics. 2013;37(2):234-42.

22. Anushiravani A, Haddadi N, Pourfarmanbar M, Mohammadkarimi V. Treatment options for nonalcoholic fatty liver disease: a double-blinded randomized placebo-controlled trial. European Journal of Gastroenterology \& Hepatology. 2019;31(5):613-7.

23. Lavine JE, Schwimmer JB, Van Natta ML, Molleston JP, Murray KF, Rosenthal $P$, et al. Effect of vitamin $E$ or metformin for treatment of nonalcoholic fatty liver disease in children and adolescents: the TONIC randomized controlled trial. JAMA. 2011:305(16):1659-68,

24. Chalasani N, Younossi Z, Lavine JE, Charlton M, Cusi K, Rinella M, et al. The diagnosis and management of nonalcoholic fatty liver disease: practice guidance from the American Association for the Study of Liver Diseases. Hepatology. 2018;67(1):328-57.

25. Sridharan K, Sivaramakrishnan G, Sequeira RP, Elamin A. Pharmacological interventions for nonalcoholic fatty liver disease: a systematic review and network meta-analysis. Postgraduate Medical Journal. 2018;94(1116):556-65.

\section{Publisher's Note}

Springer Nature remains neutral with regard to jurisdictional claims in published maps and institutional affiliations.

Ready to submit your research? Choose BMC and benefit from:

- fast, convenient online submission

- thorough peer review by experienced researchers in your field

- rapid publication on acceptance

- support for research data, including large and complex data types

- gold Open Access which fosters wider collaboration and increased citations

- maximum visibility for your research: over $100 \mathrm{M}$ website views per year

At $\mathrm{BMC}$, research is always in progress.

Learn more biomedcentral.com/submissions 